\title{
Failure of Mycoplasma pneumoniae infection to confer protection against Mycoplasma genitalium: observations from a mouse model
}

\author{
D. TAYLOR-ROBINSON and P. M. FURR \\ Imperial College School of Medicine, St. Mary's Campus, Paddington, London W2 1NY
}

\begin{abstract}
Mycoplasma pneumoniae and M. genitalium are genomically distinct but share antigens that induce some serological cross-reactivity. Therefore, the possibility that $M$. pneumoniae infection of the human respiratory tract might provide immunity to $M$. genitalium infection of the genital tract was considered. Because of the difficulty of assessing this proposition in man, it was evaluated experimentally in a mouse model. Female BALB/c mice were susceptible to infection of the vagina with $M$. pneumoniae, whereas those infected previously in the oropharynx with $M$. pneumoniae were completely immune to infection of the vagina with this mycoplasma. However, all mice with such a respiratory tract infection were susceptible to infection of the vagina with M. genitalium. The findings suggest that an $M$. pneumoniae infection of the human respiratory tract is unlikely to influence infection of the genital tract by M. genitalium.
\end{abstract}

\section{Introduction}

Mycoplasma pneumoniae is a well-known human pathogen that infects early in life, often without causing overt disease at this time [1]. Re-infection during adolescence or in later life results in a spectrum of respiratory disease, sometimes culminating in pneumonia [1]. M. genitalium, discovered two decades ago [2], is strongly associated with non-gonococcal urethritis, in a manner largely independent of Chlamydia trachomatis [3]. Although this mycoplasma has been recovered from the respiratory tract, together with $M$. pneumoniae [4], its predilection is apparently for the genital tract. M. pneumoniae and M. genitalium are genomically distinct, but they have a similar morphology, similar metabolic features and share various antigens that induce some serological cross-reactivity [5], Therefore, it is plausible that resistance to genital tract infection with $M$. genitalium might occur in man as a consequence of previous respiratory infection with $M$. pneumoniae, particularly as the latter infection is very likely to occur first.

Earlier studies in a murine model showed that experimental infection of the oropharynx with $M$. pneumoniae protects partially against re-infection of

Received 4 Sept. 2000; accepted 2 Oct. 2000.

Corresponding author: Professor D. Taylor-Robinson. the oropharynx and, more strikingly, protects completely against subsequent attempted infection of the genital tract with this mycoplasma [6]. It is also possible to infect the genital tract of mice with $M$. genitalium [7], so that this small animal model can be used to determine whether a respiratory tract infection with $M$. pneumoniae might protect the genital tract against infection with $M$. genitalium.

\section{Materials and methods}

Mice

Female BALB/c mice (6-8 weeks old) were screened for, and considered to be free of, indigenous mycoplasmas before being caged in groups of five. They were inoculated subcutaneously with progesterone (Depo-Provera; Upjohn Ltd) $2.5 \mathrm{mg}$ in a $0.1-\mathrm{ml}$ volume on four occasions, at weekly intervals [6].

\section{Media}

The glucose-containing medium used for the cultivation of $M$. pneumoniae or its isolation from murine specimens has been described previously [8], as has the medium (SP4) for the cultivation of M. genitalium [9].

\section{Experimental procedures}

Mice, anaesthetised by an intraperitoneal injection of a mixture of Hypnorm and Hypnovel, were inoculated 
intranasally with $50 \mu \mathrm{l}$ of medium containing $2.5 \times 10^{7}$ colour-changing units (ccu) of $M$. pneumoniae strain MY12763 [6]. Unanaesthetised mice were inoculated intravaginally, immediately after the second inoculation of hormone, with $50 \mu \mathrm{l}$ of medium containing the same strain of $M$. pneumoniae $\left(2.5 \times 10^{7} \mathrm{ccu}\right)$ or $M$. genitalium strain G37 $\left(2.5 \times 10^{6} \mathrm{ccu}\right)$.

Throat and vaginal specimens were collected as described previously [6], with a nasopharyngeal swab which was rotated to abrade epithelial cells, and the contents were expressed in $1.8 \mathrm{ml}$ of glucose-containing mycoplasmal broth medium. $M$. pneumoniae organisms were detected and their number estimated as described previously [6] and M. genitalium organisms were detected by means of a specific PCR assay, also as described previously [10].

\section{Results and discussion}

All of 10 hitherto uninoculated mice became infected in the vagina after intravaginal challenge with $M$. pneumoniae, the organisms persisting in large numbers $\left(10^{3}-10^{7} \mathrm{ccu}\right)$ for at least 35 days. Another 10 mice became infected in the oropharynx with $M$. pneumoniae after intranasal inoculation, the organisms persisting in small numbers $\left(10^{1}-10^{3} \mathrm{ccu}\right)$ for at least 14 days; however, none of these mice was infected in the vagina after intravaginal challenge with this mycoplasma 35 days later. In another experiment, all of 10 hitherto uninoculated mice were infected in the vagina after intravaginal challenge with $M$. genitalium, the organisms being detected up to 21 days later. Furthermore, after intranasal inoculation, 10 other mice were infected in the oropharynx with $M$. pneumoniae, in the same manner as described above. However, despite this, all of them became infected in the vagina with $M$. genitalium after intravaginal challenge with this mycoplasma 35 days later. It is not known whether there might have been a reduction in the number of $M$. genitalium organisms in the vagina of the latter mice compared to those not given $M$. pneumoniae, because the PCR assay was qualitative and not quantitative.

Mycoplasma-positive vaginal swabs probably represent infection of the cervix, as it has been shown previously by detailed examination that this site, rather than the vagina, became infected following intravaginal inoculation of M. pulmonis or M. hominis [11]. The current experiments were performed in female mice because of the difficulty of inoculating and obtaining repeated specimens from the urethra of male mice. Furthermore, the difficulty of infecting the murine respiratory tract with $M$. genitalium precluded determination of whether such infection would prevent infection of the genital tract with $M$. genitalium. However, perhaps this is an irrelevant issue in view of the apparently rare occurrence of $M$. genitalium in the human respiratory tract. Far more relevant is the fact that in the current study, and in a previous one [6], complete protection against $M$. pneumoniae infection of the murine genital tract was shown to occur as a consequence of a prior infection of the respiratory tract with $M$. pneumoniae. Immunity induced in this way is a plausible reason for $M$. pneumoniae being detected only rarely in the human genital tract [12], despite the opportunity for such infection to occur. Infection of the human repiratory tract with $M$. pneumoniae is far more common, so that the question of whether such infection might protect the genital tract against $M$. genitalium is germane. It is a question that would be difficult to resolve from an epidemiological viewpoint. Although the extent to which the findings of a study in a murine model are relevant to the human situation is debatable, the result of the current study does suggest that the induction of cross-protective immunity is unlikely.

We thank Dr Claire Gilroy for undertaking the PCR assay.

\section{References}

1. Foy HM. Infections caused by Mycoplasma pneumoniae and possible carrier state in different populations of patients. Clin Infect Dis 1993; 17 Suppl 1: 37-46.

2. Tully JG, Taylor-Robinson D, Cole RM, Rose DL. A newly discovered mycoplasma in the human urogenital tract. Lancet 1981; 1: 1288-1291.

3. Horner PJ, Gilroy CB, Thomas BJ, Naidoo ROM, TaylorRobinson D. Association of Mycoplasma genitalium with acute non-gonococcal urethritis. Lancet 1993; 342: 582-585.

4. Baseman JB, Dallo SF, Tully JG, Rose DL. Isolation and characterization of Mycoplasma genitalium strains from the human respiratory tract. J Clin Microbiol 1988; 26: 22662269.

5. Lind K. Serological cross-reactions between "Mycoplasma genitalium" and M. pneumoniae. Lancet 1982; 2: 1158-1159.

6. Furr P, Taylor-Robinson D. Colonization of the respiratory and genital tracts of female mice with Mycoplasma pneumoniae and protection afforded to the genital tract by prior respiratory colonization. Int J Exp Path 1999; 80: 35-39.

7. Furr PM, Taylor-Robinson D. Factors influencing the ability of different mycoplasmas to colonize the genital tract of hormone-treated female mice. Int J Exp Path 1993: 74: 97101.

8. Taylor-Robinson D, Furr PM. Recovery and identification of human genital tract mycoplasmas. Isr J Med Sci 1981; 17: $648-653$.

9. Tully, JG, Rose DL, Whitcomb RF, Wenzel RP. Enhanced isolation of Mycoplasma pneumoniae from throat washings with a newly modified culture medium. J Infect Dis 1979; 139: 478-482.

10. Palmer HM, Gilroy CB, Furr PM, Taylor-Robinson D. Development and evaluation of the polymerase chain reaction to detect Mycoplasma genitalium. FEMS Microbiol Lett 1991; 77: 199-204.

11. Furr PM, Sarathchandra P, Hetherington CM, Taylor-Robinson D. Site of localization of Mycoplasma pulmonis and Mycoplasma hominis in the genital tract of female mice demonstrated by culture and scanning and immuno-electron microscopy. Int J Exp Path 1995; 76: 131-143.

12. Goulet M, Dular R, Tully JG, Billowes G, Kasatiya S. Isolation of Mycoplasma pneumoniae from the human urogenital tract. $J$ Clin Microbiol 1995; 33: 2823-2825. 